\title{
Predação em girinos por uma vespa e outras associaçōes de insetos com ninhos de duas espécies de rãs da Amazônia
}

\author{
Lawrence A. Lacey (")
}

\begin{abstract}
Resumo
Fêmeas adultas do vespídeo Angiopolybia pallens (Lepeltier) foram observadas alimentando-se do material do ninho, ovos e girinos do ninho de espuma construido pela rã Leptodactylus pentadactylus (Laurenti) próximo de Manaus, Brasil. Larvas e adultos de moscas de efidrídeos, Gastrops willistoni Cresson, foram encontrados em todos os ninhos de espuma de L. pentadactylus encontrados entre 15 de julho a 21 de dezembro de 1978. As larvas provavelmente alimentamse de ovos não desenvolvidos, material do ninho e dos corpos de insetos que tinham ficado presos no material viscoso. Um eucoilídeo parasito de larvas de efidrídeo foi observado "in situ" e criado em laboratório das coletas de campo de larvas de G. willistoni. Associaçōes esporádicas adicionais são também assinaladas. Fêmeas adultas de Angiopolybia obidensis (Ducke) fo. ram observadas em uma ocasiāo comendo a gelatina ao redor dos ovos de rã (hylídeo), Osteocephalus tauri. nus Steindachner.
\end{abstract}

As associações de insetos com ovos e jovens de Anuros da Amazônia têm recebido pouca atenção. Entretanto, Bokermann (1957a) observou um efedrídeo perto de São Paulo, Brasil, identificado como Gastrops niger Williston, em ninhos de espuma de Physalaemus curvieri Fitzinger, predando os embriões. Uma observação similar foi feita no Rio de Janeiro por Bertha Lutz (apud Wirth, 1958) envolvendo G. willistoni Cresson. Observações mais detalhadas de larvas de moscas predando ovos de rãs são dadas por Villa (1977). Ele observou predaçăo por uma Drosophila não descrita nos ninhos de Centronella fleischamanni na Nica. rágua e Costa Rica. Informações adicionais de moscas associadas em ninhos de rãs são dadas por Villa (1978).

No processo de evolução de um habitat de margem d'água até terrestre, os membros do gênero Leptodactylus (Anura: Leptodactylidae) depositam seus ovos em um ninho de espuma, resultados de secreções glandulares as quais são transformadas em espumas (Heyer, 1969). Leptodactylus pentadactylus (Laurenti) usualmente constroem seus ninhos de espumas em buracos próximo à água (Breder, 1946) ou ao lado de pequenas poças (Bokermann, 1957b). Heyer (1969) cita as vantagens desta estratégia de desova como: 1 . proteçăo dos ovos por causa dos predadores da poça; 2. resistência à dessecação. A superficie pegajosa dos ninhos são adornadas com os corpos dos inse tos que ficaram grudados depois de terem entrado em contato com o material viscoso. Alguns insetos, entretanto, parecem ser adaptados a caminhar sobre a superfície e são observados como visitantes regulares de ninhos. Este estudo foi realizado para investigar as relações destes insetos com os ninhos de espuma, ovos e jovens de $L$. pentadactylus.

Observações adicionais foram feitas em associações de uma vespa com ovos de uma outra rã. Osteocephalus taurinus Steindachner (Hylidae). Esta rã normalmente deposita seus ovos em uma película superficial de pequenas poças d'água e outras depressões alagadas na floresta (Bokermann, 1964). Informações suplementares sobre o jovem de $O$. taurinus são apresentadas por Duellman \& Lescure (1973).

\section{MATERIAIS E MÉTODOS}

Catorze excursões foram realizadas entre 15 de julho e 21 de dezembro de 1978 à Reserva Ducke, uma instalação do instituto Nacio. nal de Pesquisas da Amazônia (INPA), localizada a $26 \mathrm{~km}$ de Manaus, Brasil, com o proposito de observar e coletar espécimes associados com os ninhos de espuma de L. pentaciactylus. Os ninhos de espuma e seus insetos

(") - Instituto Nacional de Pesquisas da Amazônia, Manaus. 
associados foram também fotografados para a documentação das atividades que foram observadas.

A associação de larvas de efidrídeos encontradas no ninho com adultos capturados na superfície foi confirmada pelo transporte de material do ninho de espuma para o laboratório e pela criação de larvas até adultos. Um eucoilídeo parasita foi também criado das larvas dos efidrídeos no ninho.

Observaçōes sobre a interação de um vespídeo e os ovos de $O$. taurinus foram feitas em uma só excursão $(27 / 08 / 78)$.

Espécimes testemunhas do vespídeo cole. tados neste estudo foram depositados no $\mathrm{Mu}$ seu Britânico (História Natural), no Museu Goeldi, Belém, Pará, Brasil e no Museu Entomológico do INPA, Manaus, Amazonas, Brasil. Larvas e adultos dos efidrídeos foram depositados na Smithsonian Institution, no Museu Entomológico da Universidade de Califórnia, Riverside (UCR) e no INPA; Espécimes do Eucoilídeos foram depositados na UCR e INPA.

\section{OBSERVAÇÕES E DISCUSSÃO}

Três insetos que foram comumente observados no ninho de espuma de $L$. pentadactylus: Angiopolybia pallens (Lepeltier) (Hymenoptera: Vespidae), Gastrops willistoni Cresson (Diptera: Ephydridae) e uma vespa eucoilídeo (Hymenoptera: Eucoilidae).

G. willistoni foi o mais presente dos três, sendo observado em todo ninho de espuma que foi encontrado durante o período de estudo. Freqüentemente, várias centenas de adultos foram encontrados no mesmo ninho. A atração diferencial dos ninhos comparados com a área circundante foi muito notável (Fig. 1). Quando perturbados, os adultos voavam a uma curta distância e usualmente pousavam na vegetação próxima. A maioria das moscas retornavam para o ninho dentro de poucos momentos.

Movimentos alimentares foram constantemente feitos pelos adultos quando caminhavam sobre a superfície do ninho de espuma. Cruzamentos e o que apareceu ser oviposição foram também observados sobre o ninho.
Larvas de efidrídeos foram encontradas no material do ninho de todos os 25 ninhos que foram examinados pormenorizadamente (Fig. 2). Adultos de G. willistoni foram criados em laboratório de larvas coletadas no campo.

Larvas de $(G$. willistoni foram observados em material de ninhos de espuma que foi mantido em "beakers" de $2.000 \mathrm{ml}$. Grupos de larvas foram vistos atacando embriões pouco desenvolvidos, mas baseando as observações feitas até aqui as larvas não parecem se alimentar somente dos embriões de L. pentadactylus. Na maioria dos ninhos, girinos aparentemente saudáveis e as sempre presentes larvas de $G$. willistoni foram freqüentemente encontradas desenvolvendo-se lado a lado. É provável que a alimentação das larvas poderia ser suplementada por insetos mortos que ficam imobilizados no material do ninho. Ocasionalmente, contudo, foram encontrados ninhos completamente sem girinos, os quais estavam completamente cheio de larvas de $G$. willistoni. Além da predação, densas populações de $G$. willistoni são indubitavelmente prejudiciais ao bem-estar dos girinos face à evidente produção de amônias da urina. As larvas de efidrídeos são conhecidas em uma variedades de habitats, variando desde minador de folhas e espécies aquáticas até aquelas que habitam e se alimentam de esgotos e cadáveres (Oldroyd, 1964).

O eucoilídeo foi observado em mais da metade dos ninhos que foram estudados em outubro e novembro. Diversos ninhos tinham mais de uma dúzia de fêmeas adultas na superfície ostensivamente com o propósito de ovipositar nas larvas de efidrídeos.

Eucoilídeos adultos foram criados no la. boratório de larvas, de G. willistoni, coletadas no campo confirmando a associação entre os dois. Os eucoilídeos são parasitas primários de larvas de Diptera (Clausen, 1972).

A. pallens foi observada em todas excursões. Ela foi usualmente encontrada em menos que $25 \%$ dos ninhos de espuma e de 15 de julho a 15 de outubro de 1978 , somente 1 a 2 vespas foram vistas em cada ninho. A primeira atividade que foi observada foi uma tentativa de predação de um girino por A. pallens. Quando não perturbados, os girinos de $L$. pen- 


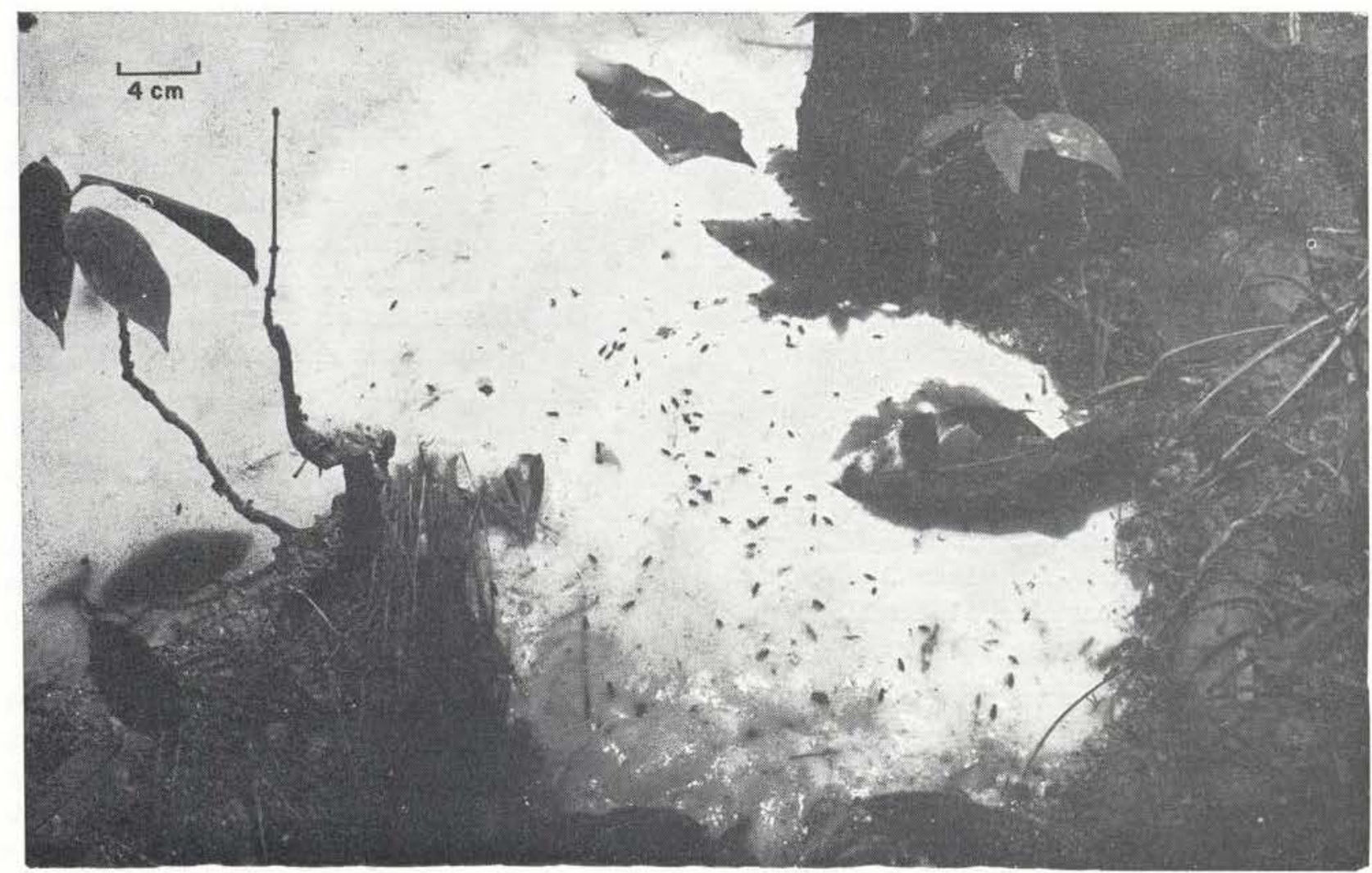

Fig. 1 - Adultos do efidrídeo Gastrops willistoni sobre o ninho de espuma de Leptodactylus pentadactylus.

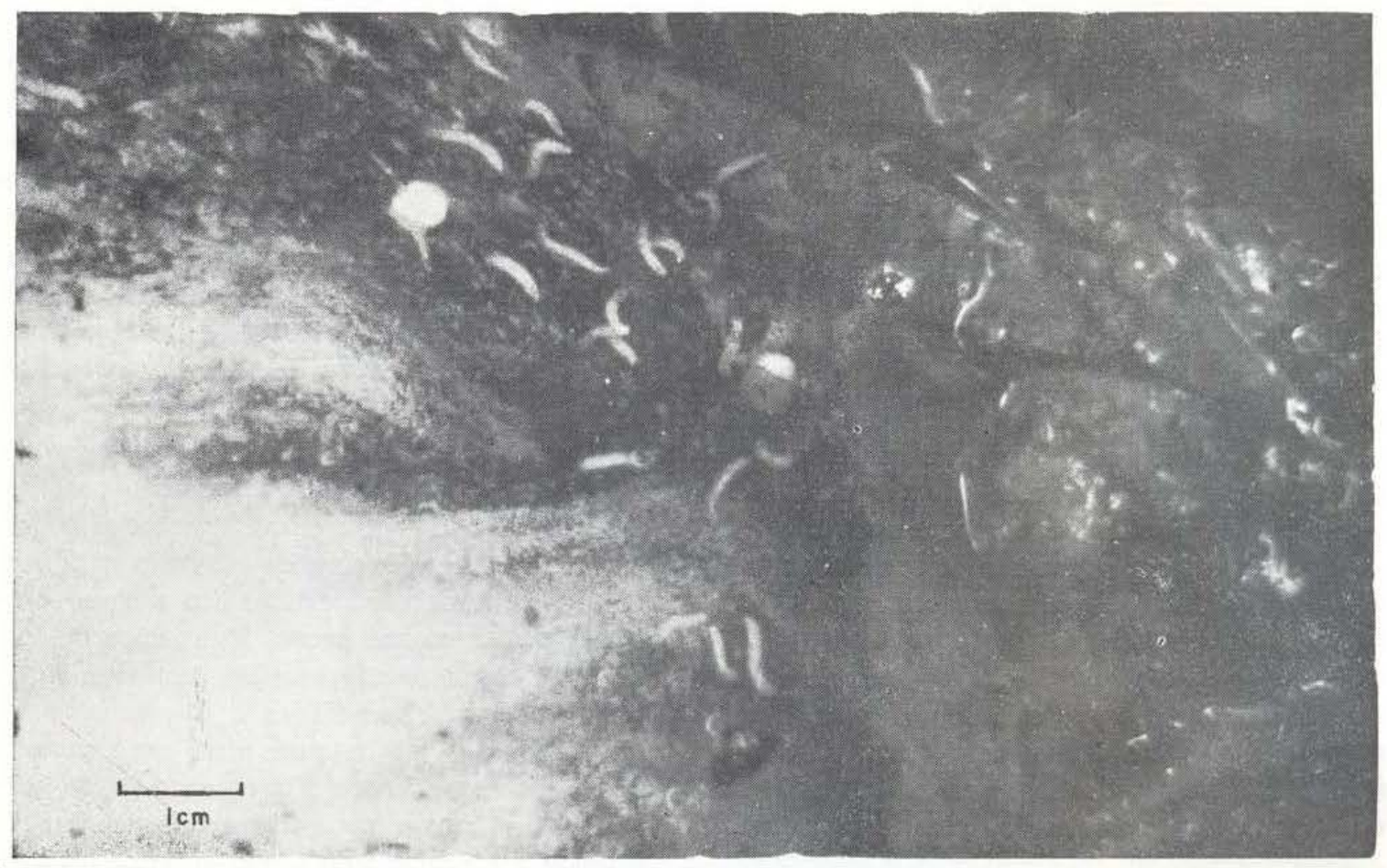

Fig. 2 - Larvas de G. willistoni no material do ninho de L. pentadactylus. Esferas brancos são ovos de L. pentadactylus. 
tadactylus permanecem imóveis na superfície do ninho. Quando uma fêmea de A. pallens pousava sobre o ninho, próximo à massa de gırınos que se movia lentamente, fazia ımeaıto contato mandibular com o girino e parecia utilizar as patas protoráxicas para segurar a presa. Simultaneamente, os girinos, e em par. vicular ,a presa subjugada nadavam para proieger-se dentro da espuma viscosa e pegajosa. Tentativas repetidas feitas pela vesça roram realizadas quando alguns dos girinos voltavam à superfície, porém sem resultadio. E duvidoso que $A$. pallens pudesse ter sido capaz de liberar o girino do ninho muito menos voar com ele mesmo que uma captura com proveito tivesse sido feita, visto que os gir. nos eram aproximadamente 1,5 até 2,0 vezes o tamanho da vespa $(1,0 \mathrm{~cm})$. Nos três meses seguintes após a primeira observação da ten. tativa de predação, as vespas foram observadas sobre o ninho de espuma em toda a Reserva, mas nenhum comportamento adicional de predação foi visto. As vespas, contudo, foram observadas reunindo o material de espu ma e procurando ativamente o material do ninho com suas mandíbulas. Quando uma boiz de material do ninho foi segura, a vespa carregou-a sob sua cabeça, segurando-a aparentemente com os palpos.

Em meados de outubro, depois de um breve período sem chuva, todos os ninhos que foram encontrados estavam parcialmente secos na superfície, dando-lhes um aspecto rugoso mas de uma consistência viscosa. Neste tempo $A$. pallens foi observado no ninho, mas suas visitas foram muito breves e não mais que 1 a 2 vespas por ninho foram vistas. De. pois a crosta foi artificialmente removida, a primeira vespa, encontrando a viscosa subsuperficie da espuma, pousou e começou a acumular e procurar a superfície da espuma como tinha sido visto nas excursões anteriores. Dentro de 20 minutos, 9 vespas agregaram-se no ninho alterado (Fig. 3). Tanto comunicação intra-colonial, estímulo olfatário (material do ninho) ou resposta visual para $A$. pallens (facilitação social) poderia ser responsável pelo rápido aumento no número de vespas no ninho. Os arredores do ninho com a superfície rugosa ainda intacta foram ainda freqüentados, brevemente por 2 ou menos A. pallens.
Quando ovos de L. pentadactylus foram encontrados por uma vespa caçadora, outras vespas sobre ninho foram atraídas para a area mais próxima dos ovos dentro de curto tempo e começaram a alimentar-se. Na Fig. 3, as 3 vespídeos na metade à direita da figura estẩo comendo o mesmo ovo. A Fig. 4 apresenta A. pallens removendo o resto final de um ovo. Note a elasticidade do material do ninho de espuma no momento em que o ovo está sendo puxado para fora.

Os vespideos pareciam menos adaptados do que os $G$. willistoni para caminhar sobre a superfície pegajosa. As asas em muitas vezes utilizadas, aparentemente para proporcionar maior altura enquanto as vespas estavam caçando e especialmente quando estavam removendo materiais do ninho.

Quando girinos jovens $(1,1 \mathrm{~cm})$ foram removidos pelo autor de dentro do ninho e colocados na superfície da espuma, eles foram atacados imediatamente (Fig. 5). Em vez de tentar voar com o girino inteiro, a cabeça e a cauda foram retiradas pelas duas vespas pouco depois da foto ter sido batida. O saco vitelínico foi então deixado de lado e carregado por outra $A$. pallens.

Em uma ocasião, durante fins de outubro, A. pallens foi observado removendo uma larva de G. willistoni, de um ninho de espuma (Fig. 6), amassando-a totalmente e então abandonando-a aparentemente. A procura de material de espuma com as mandibulas que foi observada durante excursōes anteriores pode ter sido caça às larvas de G. willistoni como também a ovos de L. pentadactylus.

Normalmente a superfície pegajosa dos ninhos de espuma fornecem proteção para os ovos e girinos, contra outros insetos. Contudo, durante duas excursões no meio e fim de outubro, quando a superfície dos ninhos estavam mais ou menos secas, 3 espécies de formigas, Crematogaster brasiliensis Mayr, Ectatomma tuberculatum (Oliv.) e Camponotus femoratum ( $F$ ) foram encontradas em razoavelmente grande número alimentando-se do material do ninho e ovos de todos os ninhos. Cuando a superfície do ninho anterior foi carregada pelas formigas, a área abaixo tornouse seca permitindo acesso mais profundo. Es. 


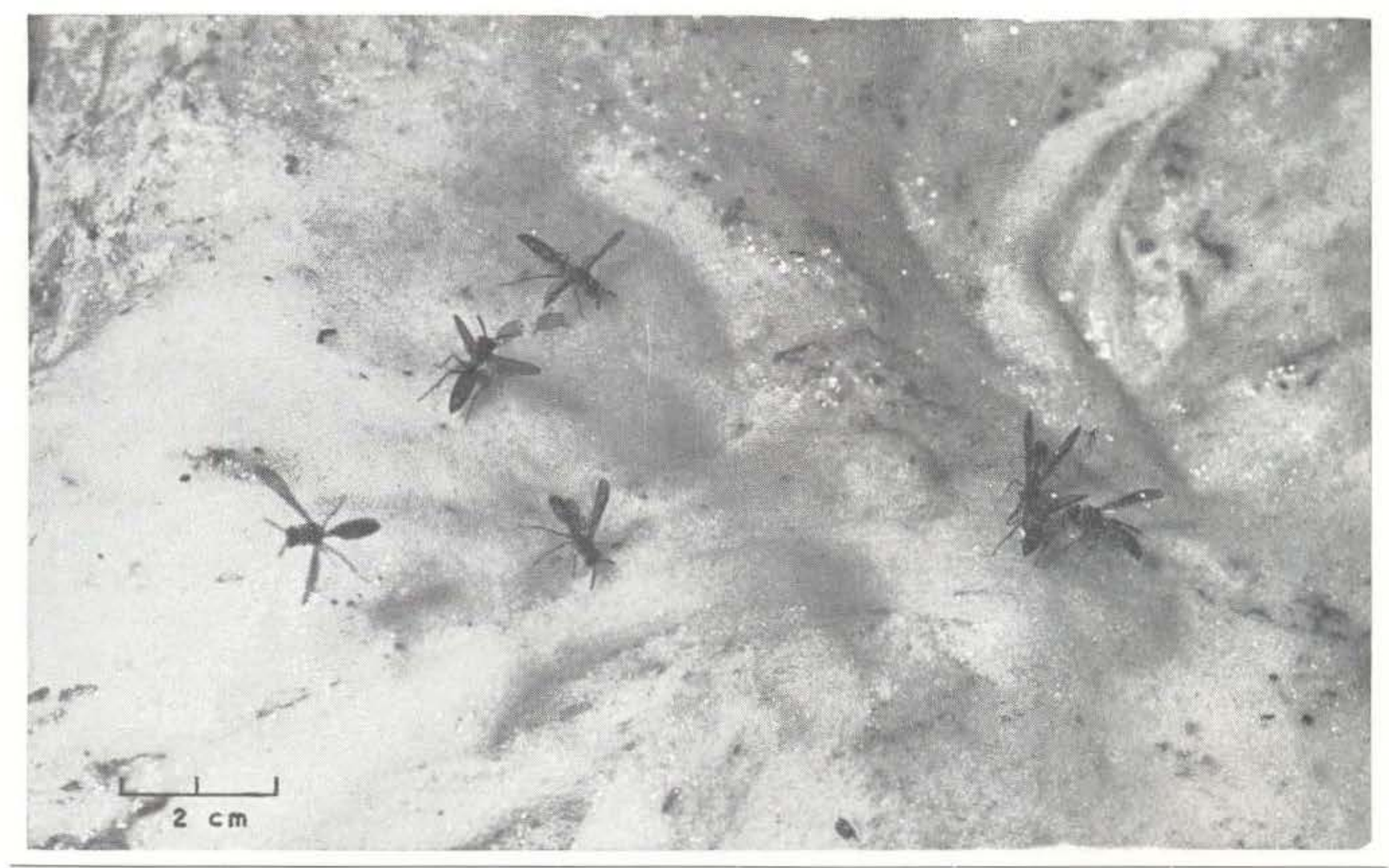

Fig. 3 - Angiopolybia pallens caçando sobre o ninho de L. pentadactylus (Foto de Bárbara Gibbs).

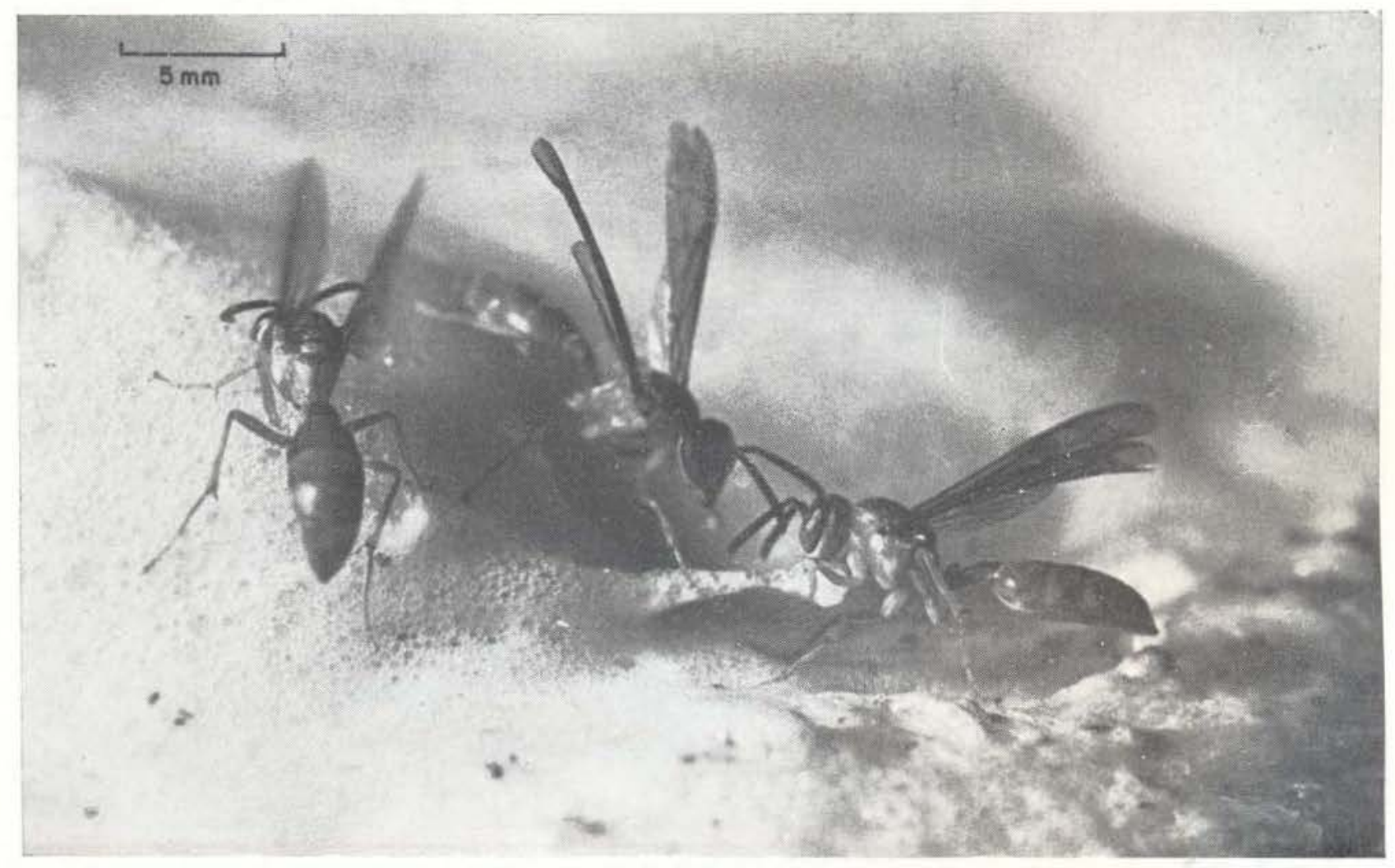

Fig. 4 - A. pallens removendo porçōes remanescentes de um ovo de L. pentadactylus (Foto de Bárbara Gibbs). 


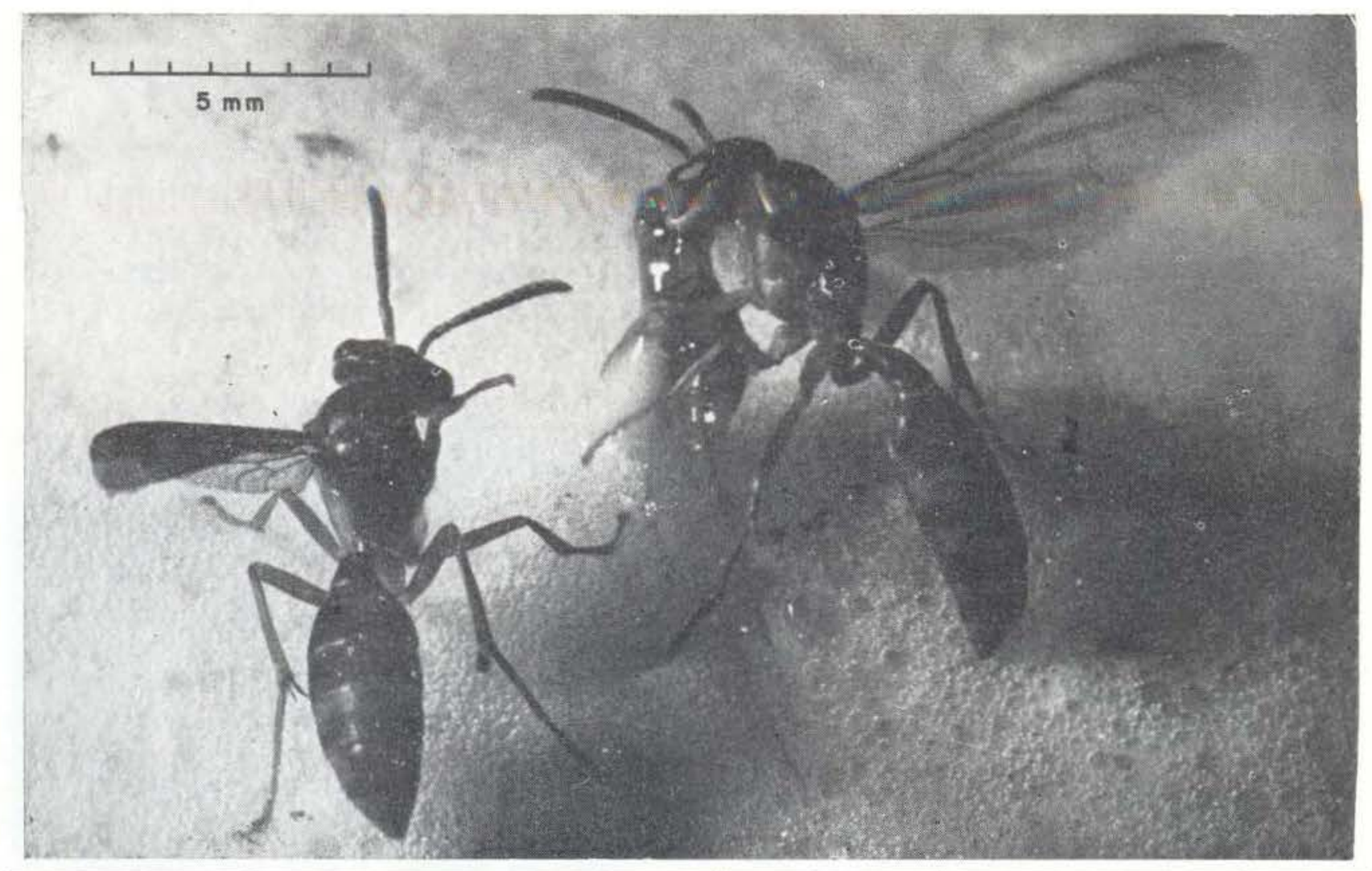

Fig. 5 - A. pallens predando um girino de L. pentadactylus (Foto de Bárbara Gibbs).

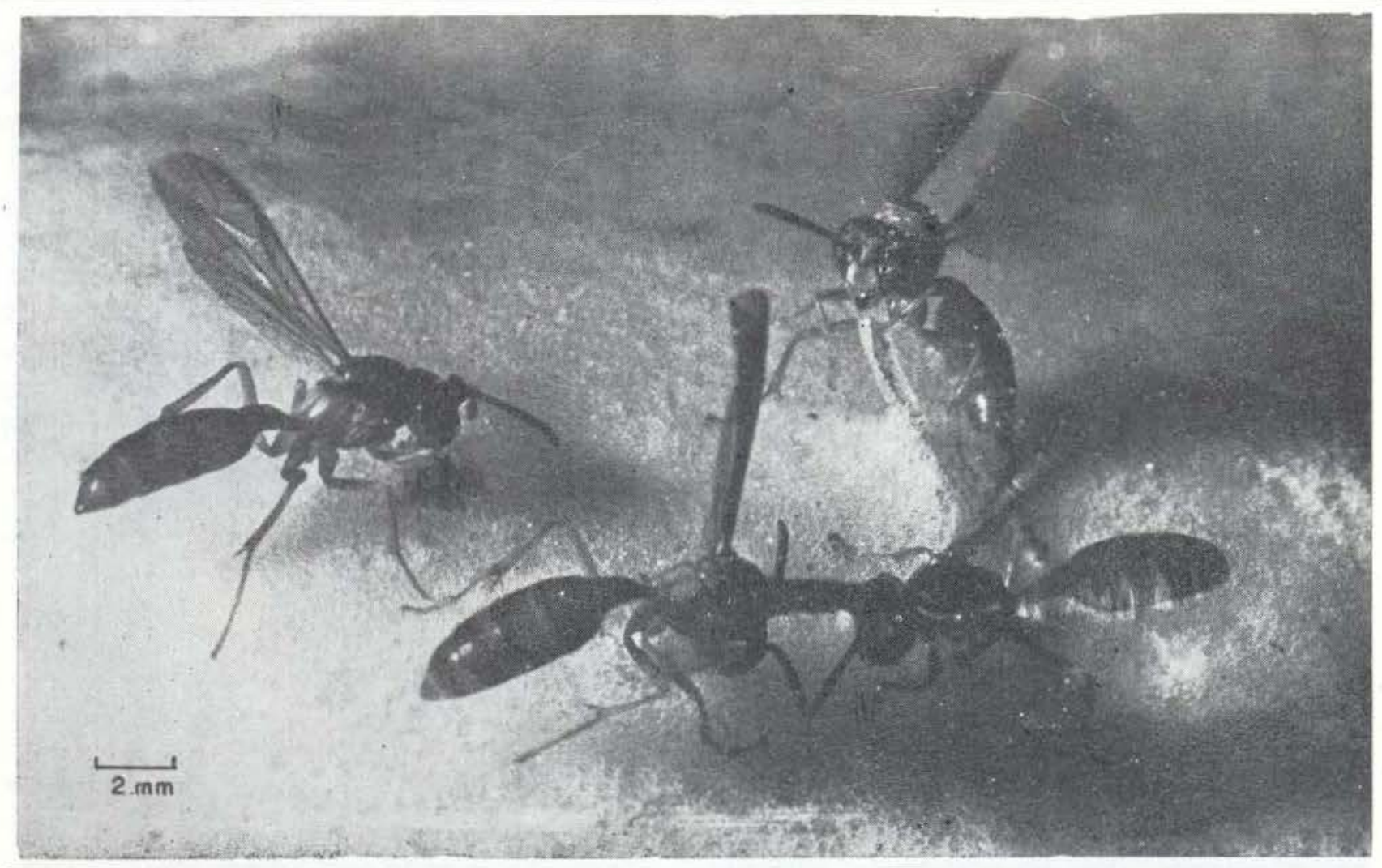

Fig, 6 - A. pallens alimentando-se no ninho de L. pentadactylus. A direita e acima, uma fêmea está removendo uma larva de G. willistoni (Foto de Bárbara Gibbs). 
te tipo de caça efetuado pelas formigas é aparentemnete uma atividade comum oportunis. ta quando a superfície do ninho se torna se$\mathrm{ca}$, o bastante para permitir a invasão. Outras espécies de formigas estão provavelmente envolvidas. Formigas operárias de $C$. temoratum eram também perseguidas por um espécie não descrito de Apocephalus (forídeo).

Dois outros insetos foram observados, de tempo em tempo, sobre os ninhos de L. pentadactylus: um não identificado colembola $e$ um velídeo. Dada a localização do ninho, sua entrada foi provavelmente incidental.

Os ovos da rã (hilídeo) $O$. taurinus foram ocasionalmente observados nas águas paradas e rasas da Reserva. Uma vez no final de agosto de 1978, o vespídeo Angiopolybia obidensis (Ducke) foi visto removendo, determinados ovos de $O$. taurinus da água rasa e comendo parte da gelatina em torno do embrião. A porção restante da gelatina e o embrião foram deixados à margem da poça. Mais que uma dúzia de $A$. obidensis foi observada na poça, cada um alimentando-se de diversos ovos. Depois de uma refeição de gelatina, as vespas, muitas vezes, voavam para a vegetação próxima e ficavam por um ou dois minutos, retornando em seguida à poça. Esse comportamento não foi observado outra vez durante o resto do estudo.

Vespídeos são conhecidos geralmente como predadores de insetos e de outros invertebrados (Akre \& Davis, 1978). Esta familia tem sido citada também como se alimentando de carne fresca de carcaças de vertebrados (Payne \& Masson 1971, Green et al., 1976) como também atacando e alimentando-se de pássaros vivos (Wild, 1927 e Grant, 1959, apud Akre et al., 1974; Spradberry, 1974), humanos (Phipps, 1974 e Akre, comunicação pessoal) e cavalos (Hawtorne, 1974). Contudo a predação sobre ovos e girinos de L. pentadactylus por A. pallens e a alimentação de ovos por A. obidensis é aparentemente o primeiro registro da predação de vespídeo sobre Anuros vivos.

\section{Agradectmentos}

Estou profundamente agradecido pela ajuda prestada pelo Dr. Walter Hödl, Universida- de de Viena, pela identificação das espécies de rã, informações e referências da biologia de rãs. A ajuda pela identificação de associação de insetos pelo Sr. W. L. Overal, Museu Goeldi (Vespidae e Formicidae) e Dr. W. Mathis, Instituto Smithsonian (Ephydridae) é também reconhecida. Estou especialmente grato ao Dr. R. D. Akre, Universidade Estadual de Washington e Sr. S. I. Frommer, Universidade da Califórnia por sua revisăo no manuscrito e pelo fornecimento de literatura. Minha gratidão estende-se a Sra. Bárbara Gibbs por doar suas fotografias e pelo seu empenho e habilidade na datilografia do manuscrito.

\section{SUMMARY}

Adult females of the vespid Angiopolybia pailens (Lepeltier) were observed feeding upon nest material, eggs, and tadpoles of the foam nest building frog. Leptodactylus pentadactylus (Laurenti) near Manaus, Brasil. Larvae and adults of the ephydrid fly, Gastrops willistoni Cresson were found at every foam nest of L. pentadactylus that was encountered between Júly 15 and Dec. 21, 1978. The larvae probably feed on the undeveloped eggs, nest material and on the bodies of insects that have become stuck in the viscous nests. An eucoilid parasite of the ephydrid larvae was observed in situ and was reared in the laboratory from field collected larvae of G. willistoni. Additional sporadic associates are also listed. Adult females of Angiopolybia obidensis (Ducke) were observed on one occasion eating the gelatin from around the eggs of the hylid frog. Osteocephaius taurinus Steindachner.

\section{BIBLIOGRAFIA}

AKRE, R.D. \& DAVIS, H.G.

1978 - Biology and pest status of venomous wasps. Ann. Rev. Entomol., 23: 215-238.

AKRe, R.D.; MAC DONALD, J.F. \& Hill, W.B

1974 - Yellow-jacket literature (Hymenoptera : Vespidae). Melanderia, 18: 67-93.

BOKERMANN, W.C.A

1957a- Frog eggs parasitized by dipterous larvae Herpetologia 13: 231-232.

$1957 \mathrm{~b}$ - Notas sobre a biologia de Leptodactylus flavopictus Lutz, 1926. Rev. Brasil. Biol. 17: $495-500$

1964 - Field observations on the hylid frog Osteocephalus taurinus Fitz. Herpetologica, 20: 252-255.

BREDER, C.M., JR.

1946 - Amphibians and reptiles of the Rio Chucunaque drainage, Darién, Panamá, with notes on their life histories and habits. Bull. Aber. Mus. Nat. Hist., 86: 375-436. 
Clausen, C.P.

1972 - Entomophagous Insects. Hafner, New York $688 \mathrm{pp}$.

Duellman, W.E. \& Lescure, J.

1973 - Life history and ecology of the hylid frog Osteocephalus taurinus, with observations on larval behavior. Occ. Pap. Mus. Nat. Hist. Univ. Kan., 13: 1-12.

GRANT, J.

1959 - Hummingbirds attacked by wasps. Can . Fld. Nat, $73: 174$.

Greene, A.; Akre, R.D. \& Landolt, P.

1976 - The aerial yellow-jacket, Dolichovespula arenaria (Fab.): nesting biology, reproductive production, and behavior (Hymenoptera: Vespidae)). Melandria, 26: 1-34.

HAWTHORNE, R.M.

1974 - Cooperative economic insect report for California for the week ending Oct 18, 1974. (Mimeografado).

HEYER, W.R.

1969 - The adaptive ecology of the species groups of the genus Leptodactylus (Amphibia, Leptodactylidae). Evolution, 23: 421-428.

OLDROYD, $\mathrm{H}$.

1964 - The Natural History of Flies. W. W. Norton, New York. 324 pp.
PAYNE, J.A. \& MASON, W.R.M.

1971 - Hymenoptera associated with pig carrion Ent. Soc. Wash., 73: 132-141.

PhIPPS, J.

1974 - The vampire wasps of British Columbia. Bull. Ent. Soc, Can., 6: 134.

SPRADBERY, J.P.

1973 - Wasps : An account of the biology and ne. tural history of solitary and social wasps. Univ Wash, Press Seattle, 408 pp.

VILLA, J.

1977 - A symbiotic relationship between frog (Amphibia, Anura, Centrolenidae) and fly larvae (Drosophilidae). J. Herpetol., 11: 317-322.

1978 - Symbiotic relationship of the developing frog embryo, with special reference to fly larvae. $\mathrm{PhD}$ dissertation, Cornell Univ. $199 \mathrm{pp}$.

WILD, D.H.

1927 - Wasps destroying young birds. Br. Birds, 20: 254.

WIRTH, W.W.

1958 - A review of the genus Gastrops Williston, with descriptons of two new species. Proc. Ent. Soc. Wash., 60: 247-250.

(Aceito para publicação em 05/07/79) 